\title{
Relative validation of a quantitative FFQ for use in Brazilian pregnant women
}

\author{
Patrícia Barbieri ${ }^{1}$, Renata Y Nishimura ${ }^{1}$, Lívia C Crivellenti ${ }^{1}$ and Daniela S Sartorelli ${ }^{1,2, *}$ \\ 'Post-Graduate Program in Community Health, School of Medicine of Ribeirão Preto (FMRP), University of São \\ Paulo (USP), Ribeirão Preto, SP, Brazil: ${ }^{2}$ Department of Social Medicine, School of Medicine of Ribeirão Preto \\ (FMRP), University of São Paulo (USP), Avenida Bandeirantes 3900, Ribeirão Preto, SP, CEP 14049-900, Brazil
}

Submitted 31 January 2012: Final revision received 27 June 2012: Accepted 3 July 2012: First published online 16 August 2012

\begin{abstract}
Objective: To evaluate the ability of an FFQ, designed for use in Brazilian pregnant women, to estimate nutrient intakes during pregnancy.

Design: A prospective study was conducted among 103 pregnant women attended by the Brazilian national health-care service. Food intake during pregnancy was evaluated by three $24 \mathrm{~h}$ dietary recalls $(24 \mathrm{hR})$, one per trimester of pregnancy, and also by two FFQ. The FFQ with eighty-five food items included questions about frequency of intake and portion sizes during two periods: the first 24 weeks of pregnancy and the pregnancy period as a whole. Deattenuated Pearson's correlation coefficients and joint classification into quartiles of nutrient intake were applied.

Setting: Ribeirão Preto, São Paulo State, Brazil.

Subjects: One hundred and three pregnant women, aged 18-35 years.

Results: Acceptable correlation coefficients $(r>0 \cdot 35)$ were found for $\mathrm{Ca}, \mathrm{K}, \mathrm{Zn}$, $\mathrm{Mg}$, fibre, vitamin C, niacin and folic acid for intake for the first 24 weeks; and for energy, lipids, protein, carbohydrate, Fe, K, Zn, fibre, vitamin $\mathrm{B}_{6}$, riboflavin and niacin for the gestational period as a whole. A high proportion of study participants $(\geq 70 \%)$ were categorized into the same or adjacent quartiles for estimated energy, carbohydrate, $\mathrm{Ca}, \mathrm{K}$, fibre, Zn, cholesterol, vitamin A, riboflavin, niacin, vitamin $\mathrm{C}$, vitamin $\mathrm{E}$ and folic acid. Gross misclassification ranged from $2 \cdot 3 \%$ (dietary fibre) to $12 \cdot 5 \%$ (vitamin A, thiamin and SFA).

Conclusions: The FFQ is a useful tool for assessing categories of nutrient intake during pregnancy, since a high proportion of women were classified into the same or adjacent quartiles.
\end{abstract}

Keywords

FFQ

Pregnant women Validation studies
Inadequate maternal diet is a relevant risk factor for complications during pregnancy ${ }^{(1,2)}$, impaired fetal development $^{(3)}$ and may also be linked to higher risk of chronic diseases during childhood ${ }^{(4)}$ and adult life ${ }^{(5)}$.

The FFQ is a method widely used in epidemiological studies aimed at categorizing individuals into different levels of consumption and determining their relationships with health outcomes ${ }^{(6,7)}$. The FFQ's ability to assess dietary intake of pregnant women is due to the fact this method spans a longer time frame, thus allowing the detection of changes in dietary intake during each trimester of pregnancy ${ }^{(8-11)}$.

Validation studies of FFQ for use in pregnant women have shown that nutrient estimates using this method exhibit high levels of agreement with intake estimates using the $24 \mathrm{~h}$ dietary recall $(24 \mathrm{hR})^{(12-14)}$ or daily records $(\mathrm{DR})^{(15,16)}$, suggesting a good accuracy of the FFQ for assessing dietary intake in this population group. Since $24 \mathrm{hR}$ and FFQ might have correlated errors, related to memory and estimation of portion size, the use of multiple DR or weighed records as the reference method is recommended. Nevertheless, when literacy or motivation of participants is low, the $24 \mathrm{hR}$ might be applied as the reference measure of nutrient intake ${ }^{(6)}$.

In an earlier study, an FFQ was developed for use in pregnant women attended by the national health-care system in the municipality of Ribeirão Preto, São Paulo State, Brazil $^{(17)}$. The aim of the present study was to verify the relative validity of this FFQ against the $24 \mathrm{hR}$ instrument for estimating nutrient intakes over the three trimesters of pregnancy. The FFQ will subsequently be employed in an investigation exploring the relationship between dietary intake at the beginning of the pregnancy and the occurrence of gestational diabetes mellitus. However, given the lack of FFQ developed and validated specifically for use in pregnant women in Brazil, coupled with the growing body of evidence pointing to the relationship between maternal dietary intake during pregnancy and maternal and fetal outcomes, it was deemed important to also assess the accuracy of the method for estimating nutrient intakes throughout the pregnancy period as a whole. 
Accordingly, the reference periods adopted for assessing food intake were the first 6 months of pregnancy plus the pregnancy period as a whole.

\section{Method}

\section{Study design and population}

A prospective study involving 103 pregnant women users of the Brazilian national health-care system from the municipality of Ribeirão Preto, São Paulo, was conducted. The municipality is located $313 \mathrm{~km}$ from São Paulo, the State capital, and has an estimated population of 583842 inhabitants $^{(18)}$. A convenience study sample was employed and sample size was determined based on the recommendation that 100 individuals would be required to assess the agreement between the two methods used for assessing dietary intake ${ }^{(6)}$.

The study inclusion criteria were: (i) age 18-35 years; (ii) pre-gravid BMI of $18 \cdot 5-25 \cdot 0 \mathrm{~kg} / \mathrm{m}^{2(19)}$; (iii) gestational age of up to 14 weeks at first study interview; and (iv) absence of pathologies which may change the pattern of food consumption during pregnancy such as diabetes, cardiopathies, nephropathies and hypertension.

Data collection was carried out at four basic health clinics situated in southern, eastern and western regions of the municipality by previously trained nutritionists. Participants were assessed at study baseline (up to 14th week of gestation), at the second trimester (between 14th and 28th weeks) and again at the third trimester (after 28th week of gestation). The first study assessment was carried out during the first antenatal visit of the pregnant woman between September 2009 and May 2010. The second and third assessments were performed at antenatal check-ups or during homecare visits.

\section{Sociodemographic characteristics}

Data on age, schooling, socio-economic status and presence of morbidities were collected using a structured questionnaire applied at the first study assessment. Economic status was classified according to the Brazilian Criteria for Economic Classification (CCEB) based on items owned by and educational level of the head of the family ${ }^{(20)}$.

\section{Assessment of nutritional status and gestational age}

Gestational age was calculated based on the date of last menstruation and on ultrasound scan. Pre-gravid weight was reported by the pregnant woman and corrected according to data contained in medical charts. The criteria of the US Institute of Medicine ${ }^{(19)}$ were employed to calculate the adequacy of pre-gravid BMI.

\section{Quantitative FFQ}

The FFQ was previously devised for pregnant women attended by the basic health units of Ribeirão Preto, and its methodology is described elsewhere ${ }^{(17)}$. Briefly, a 24hR was obtained from 150 pregnant women (fifty for each trimester of pregnancy). A second $24 \mathrm{hR}$ was obtained in a sub-sample of ninety pregnant women in order to correct for within-person variance. The food list was systematically shortened by stepwise multiple regression analysis, using the deattenuated energy-adjusted nutrients of interest as dependent variables. A total of eighty-five foods were identified which explained the greatest between-person variance of intake of each nutrient (between $74 \%$ and 99\%). The 25th, 50th, 75th and 100th percentiles were employed to determine small, medium, large and extra-large portion sizes, respectively. The reproducibility of the FFQ was tested in a previous study conducted among ninety-five pregnant women. Two FFQ were obtained spaced between 15 and $45 \mathrm{~d}$ apart. The intra-class correlation of unadjusted nutrients was $0 \cdot 81$, after adjustment for energy the mean correlation was $0 \cdot 70$. Joint classification of energy-adjusted nutrient intakes into the same or adjacent quartiles for the two FFQ was $82 \%$, and less than $8 \%$ of women were misclassified (data not published).

In the present study, the FFQ was applied at the second (between 14th and 28th weeks of gestation) and third (after 28th week of gestation) study assessments. Participants were asked to report the frequency of each food item since the establishment of the pregnancy.

\section{Nutrient intake estimated by $24 \mathrm{~b}$ dietary recalls}

Three $24 \mathrm{hR}$ were obtained from all study participants, corresponding to one recall per trimester of pregnancy. The $24 \mathrm{hR}$ were administered by a trained nutritionist employing the multiple-pass technique ${ }^{(21)}$. Conversion of household measures into grams of food consumed was carried out with the aid of Brazilian reference manuals containing data on recipes and household measures ${ }^{(22,23)}$.

The nutrients of interest in the present study were: energy, carbohydrate, protein, lipids, Fe, Ca, K, vitamin A, thiamin, riboflavin, niacin, vitamin $\mathrm{C}$, SFA, cholesterol, folic acid, fibre, vitamin $\mathrm{E}, \mathrm{Zn}$, vitamin $\mathrm{B}_{6}, \mathrm{Mg}$ and $\mathrm{Cu}$.

Nutrient composition of the FFQ estimate was analysed using the Dietsys ${ }^{\circledR}$ program (HHHQ DietSys Analysis Software version $4 \cdot 02$; National Cancer Institute, Bethesda, MD, USA, 1999). The nutritional composition of the recalls was analysed by the NutWin ${ }^{\circledR}$ program (NutWin Software, Nutrition Support Program, version 1.5; Escola Paulista de Medicina, São Paulo, São Paulo State, Brazil, 2002). Food composition data were drawn from the Brazilian table of food composition ${ }^{(24)}$ in conjunction with the US Department of Agriculture's National Nutrient Database for Standard Reference ${ }^{(25)}$.

\section{Data analysis}

Means and standard deviations were computed for continuous data on sociodemographic, nutritional status and lifestyle variables, and frequencies for categorical variables. 
Median values and interquartile ranges were obtained for the description of FFQ and 24hR nutrient estimates for the first two trimesters and for the whole gestational period. The normality of dietary variables was verified using the Kolmogorov-Smirnov test. Variables found to have a nonnormal distribution were transformed by natural logarithm prior to statistical analyses. Nutrient intakes were adjusted for total energy intake by the residual method ${ }^{(26)}$.

The energy-adjusted, deattenuated Pearson's correlation coefficient was employed to validate the FFQ. The energy-adjusted Pearson's correlation coefficient was deattenuated using the formula: $\left[1+\left(\sigma_{\text {intra }}^{2} / \sigma_{\text {inter }}^{2}\right) / n\right]^{1 / 2}$, where $n$ is the number of $24 \mathrm{hR}$ replicates, $\sigma_{\text {intra }}^{2}$ represents the within-person variance and $\sigma_{\text {inter }}^{2}$ is the betweenperson variance among the $24 \mathrm{hR}^{(27)}$.

The validation of the FFQ was also assessed by analysing the agreement between the two methods using cross-classification into quartiles of estimated energy and nutrients, and quadratic weighted kappa. Quadratic weighted $\boldsymbol{\kappa}$ is considered an appropriate test to estimate agreement when three or more categories on ordinal scales are provided ${ }^{(28)}$.

All statistical analyses were performed using the SPSS statistical software package version $17 \cdot 0$.

\section{Ethical aspects}

The project was previously approved by the Research Ethics Committee of the Centro de Saúde Escola of the Ribeirão Preto School of Medicine, University of São Paulo (protocol no. 239) and authorized by the Municipal Secretariat for Health of Ribeirão Preto. The participants agreed to take part in the study by signing a free and informed consent form.

\section{Results}

A total of 247 pregnant women were contacted between September 2009 and May 2010. Of this group, five (2\%) declined to take part in the study and 139 (56\%) were excluded for not meeting the study criteria as follows: sixty-two (25\%) due to age, forty-five (18\%) due to inadequate pre-gravid nutritional status and thirty-two (13\%) women for having advanced gestational age. This gave a total of 103 pregnant women for study.

Table 1 depicts the sociodemographic characteristics, nutritional status and lifestyle characteristics of the pregnant women at study baseline. The majority of pregnant women assessed had a schooling level of at least 8 years, belonged to economic class $\mathrm{C}$ or $\mathrm{D}+\mathrm{E}$, and were married.

Of the 103 pregnant women interviewed at study baseline (up to 14th week of gestation), eighty-eight (85\%) underwent the second assessment (carried out between 14 th and 24 th weeks of gestation) and seventytwo $(70 \%)$ took part in the third evaluation (between 25 th and 36th weeks of gestation). Among the fifteen
Table 1 Sociodemographic characteristics, nutritional status and lifestyle of pregnant women at baseline - Ribeirão Preto, São Paulo, Brazil, 2009 ( $n$ 103)

\begin{tabular}{lcc}
\hline Characteristic & Mean & SD \\
\hline Age (years) & 24 & $4 \cdot 5$ \\
Weeks of gestation & 11 & $2 \cdot 1$ \\
Pre-gravid BMI $\left(\mathrm{kg} / \mathrm{m}^{2}\right)$ & $21 \cdot 9$ & $1 \cdot 9$ \\
Monthly family income (\$US) & 680 & 340 \\
& $n$ & $\%$ \\
\cline { 2 - 3 } Education (years of schooling) & & \\
Up to 4 & 16 & $15 \cdot 7$ \\
From 4 to 8 & 34 & $33 \cdot 3$ \\
More than 8 & 52 & $51 \cdot 0$ \\
Socio-economic class & \\
A+B & 8 & $7 \cdot 8$ \\
C & 73 & $70 \cdot 9$ \\
D+E & 22 & $21 \cdot 4$ \\
Head of family & & \\
Partner/husband & 72 & $69 \cdot 9$ \\
Myself & 11 & $10 \cdot 7$ \\
Other & 20 & $19 \cdot 4$ \\
Never consume alcoholic beverages & 87 & $84 \cdot 5$ \\
Smoking during pregnancy & 15 & $14 \cdot 6$ \\
Use of folic acid & 44 & $50 \cdot 0$ \\
Usually practise physical activities & 17 & $22 \cdot 7$ \\
\hline
\end{tabular}

*Brazilian Criteria for Economic Classification (CCEB) ${ }^{(20)}$.

drop-outs at the second assessment, ten had miscarriages, two had moved to other cities and three were not found. Of the other sixteen drop-outs at the third study assessment, three had moved to other cities, five had premature delivery and eight were not found. Mean (SD) gestational weight gain between first and second interviews was 5.2 (SD $2 \cdot 8) \mathrm{kg}$, and between first and third interviews was $9 \cdot 0$ (sD 3.1$) \mathrm{kg}$. The average time interval between first and second interviews was $82 \mathrm{~d}$ (approximately 12 weeks) and between second and third interviews was $47 \mathrm{~d}$ (approximately 7 weeks).

Table 2 shows median values for nutrient intakes based on the FFQ and $24 \mathrm{hR}$ instruments. Estimated energy intake was higher on the FFQ than on the $24 \mathrm{hR}$ for both periods. Considering the nutrient estimates for the first 24 weeks of gestation, adequate crude Pearson's correlations $(r \geq 0 \cdot 4)$ were found for $\mathrm{Ca}$, vitamin $\mathrm{B}_{6}$ and folic acid. Borderline correlations $(r>0.35$ and $<0.40)$ were verified for $\mathrm{Fe}, \mathrm{K}, \mathrm{Mg}$, fibre, SFA, vitamin A, thiamin, riboflavin, niacin and vitamin C. After adjustment for energy and deattenuation, good correlations were observed for $\mathrm{Ca}, \mathrm{K}$, $\mathrm{Zn}, \mathrm{Mg}$, fibre and folic acid, and borderline correlations were found for niacin and vitamin C.

Considering estimated nutrient intakes for the gestational period as a whole, adequate unadjusted correlations were found for energy and carbohydrate, and borderline correlations for lipids, Fe, niacin and fibre. After adjustments, good correlations were found for energy, carbohydrate, niacin and fibre, and borderline correlations were observed for protein, lipids, $\mathrm{Fe}, \mathrm{K}, \mathrm{Zn}$, riboflavin and vitamin $\mathrm{B}_{6}$ (Table 2). 
Table 2 Estimated energy and nutrient intakes (median and IQR) from the FFQ and 24hR and Pearson's correlation coefficient in pregnant women - Ribeirão Preto, São Paulo, Brazil, 2009

\begin{tabular}{|c|c|c|c|c|c|c|c|}
\hline \multirow[b]{2}{*}{ Nutrient } & \multicolumn{2}{|c|}{$24 \mathrm{hR}$} & \multicolumn{2}{|c|}{ FFQ } & \multicolumn{3}{|c|}{ Pearson's correlation } \\
\hline & Median & IQR & Median & IQR & Crude & Energy-adjusted & Deattenuated $^{*}$ \\
\hline & \multicolumn{7}{|c|}{ Estimated nutrient intakes and accuracy of FFQ for first two trimesters of pregnancy $(n 88)$} \\
\hline Energy $(\mathrm{kJ} / \mathrm{d})$ & 8472 & 4749,10635 & 10740 & 8058,14581 & $0 \cdot 27$ & - & $0 \cdot 29 t$ \\
\hline Protein $(g / d)$ & 81 & 65,97 & 98 & 73,130 & 0.25 & $0 \cdot 31$ & 0.27 \\
\hline Lipids (g/d) & 61 & 42,78 & 68 & 49,97 & $0 \cdot 29$ & $0 \cdot 32$ & $0 \cdot 32$ \\
\hline Carbohydrate (g/d) & 267 & 219,333 & 364 & 274,503 & $0 \cdot 28$ & 0.25 & $0 \cdot 31$ \\
\hline $\mathrm{Ca}(\mathrm{g} / \mathrm{d})$ & 561 & 386,823 & 950 & 656,1264 & 0.51 & 0.51 & 0.57 \\
\hline $\mathrm{Fe}(\mathrm{mg} / \mathrm{d})$ & 8 & 6,10 & 10 & 7,15 & 0.35 & $0 \cdot 13$ & $0 \cdot 14$ \\
\hline$K(g / d)$ & 2272 & 1743,2754 & 3280 & 2577,4185 & $0 \cdot 36$ & $0 \cdot 37$ & 0.45 \\
\hline $\mathrm{Zn}(\mathrm{g} / \mathrm{d})$ & 10 & 8,14 & 13 & 9,18 & $0 \cdot 33$ & $0 \cdot 39$ & 0.44 \\
\hline $\mathrm{Mg}(\mathrm{mg} / \mathrm{d})$ & 210 & 162,255 & 282 & 239,380 & $0 \cdot 35$ & 0.45 & 0.53 \\
\hline $\mathrm{Cu}(\mathrm{mg} / \mathrm{d})$ & 0.8 & $0 \cdot 7,1 \cdot 2$ & $1 \cdot 5$ & $1 \cdot 1,2 \cdot 1$ & $0 \cdot 20$ & $0 \cdot 17$ & $0 \cdot 20$ \\
\hline Folic acid $(\mu \mathrm{g} / \mathrm{d})$ & 144 & 101,195 & 247 & 190, 345 & 0.42 & $0 \cdot 37$ & 0.44 \\
\hline Vitamin A (IU) & 5013 & 2937, 9024 & 10320 & 7543,16567 & $0 \cdot 31$ & $0 \cdot 29$ & 0.34 \\
\hline Vitamin A (RE) & 653 & 339,1134 & 1481 & 938,2111 & $0 \cdot 37$ & 0.28 & 0.32 \\
\hline Thiamin (mg/d) & $1 \cdot 1$ & $0.8,1.5$ & $1 \cdot 8$ & $1 \cdot 3,2 \cdot 8$ & $0 \cdot 35$ & $-0 \cdot 10$ & $-0 \cdot 10$ \\
\hline Riboflavin (mg/d) & $1 \cdot 4$ & $1 \cdot 1,2 \cdot 1$ & $2 \cdot 1$ & $1 \cdot 5,3 \cdot 0$ & $0 \cdot 40$ & 0.27 & $0 \cdot 30$ \\
\hline Niacin $(\mathrm{mg} / \mathrm{d})$ & 18 & 13,21 & 21 & 16,31 & $0 \cdot 38$ & $0 \cdot 31$ & $0 \cdot 40$ \\
\hline Vitamin C (mg/d) & 75 & 29,163 & 175 & 96,303 & $0 \cdot 40$ & $0 \cdot 40$ & $0 \cdot 40$ \\
\hline Vitamin E (mg/d) & 5 & 3,7 & 7 & 5,10 & $0 \cdot 27$ & $0 \cdot 20$ & $0 \cdot 20$ \\
\hline Vitamin $B_{6}(\mathrm{mg} / \mathrm{d})$ & 0.9 & $0 \cdot 7,1 \cdot 3$ & $1 \cdot 2$ & $0 \cdot 8,1 \cdot 6$ & 0.46 & 0.28 & 0.34 \\
\hline Fibre $(g / d)$ & 20 & 15,26 & 32 & 22,40 & $0 \cdot 36$ & 0.52 & 0.60 \\
\hline SFA $(g / d)$ & 20 & 14,27 & 26 & 10,36 & $0 \cdot 38$ & 0.25 & $0 \cdot 30$ \\
\hline Cholesterol (g/d) & 220 & 173,293 & 249 & 166,320 & $0 \cdot 30$ & $0 \cdot 25$ & $0 \cdot 30$ \\
\hline \multirow[t]{2}{*}{ Mean } & - & & - & & $0 \cdot 33$ & $0 \cdot 25$ & 0.35 \\
\hline & \multicolumn{7}{|c|}{ Estimated nutrient intakes and accuracy of FFQ for whole gestational period ( $n$ 72) } \\
\hline Energy (kJ/d) & 8539 & 6920,10719 & 11075 & 8154,13581 & 0.48 & - & $0.53 t$ \\
\hline Protein $(\mathrm{g} / \mathrm{d})$ & 83 & 67,98 & 102 & 73,130 & 0.34 & 0.27 & $0 \cdot 37$ \\
\hline Lipids (g/d) & 62 & 43,79 & 74 & 46,98 & $0 \cdot 37$ & $0 \cdot 16$ & 0.40 \\
\hline Carbohydrate $(\mathrm{g} / \mathrm{d})$ & 270 & 225,332 & 382 & 292,466 & 0.53 & $0 \cdot 17$ & 0.58 \\
\hline $\mathrm{Ca}(\mathrm{g} / \mathrm{d})$ & 607 & 469,863 & 967 & 739,1241 & $0 \cdot 26$ & 0.33 & $0 \cdot 28$ \\
\hline $\mathrm{Fe}(\mathrm{mg} / \mathrm{d})$ & 8 & 7,11 & 10 & 8,15 & 0.35 & 0.06 & 0.38 \\
\hline$K(g / d)$ & 2344 & 1848,2891 & 3385 & 2531,4037 & $0 \cdot 32$ & $0 \cdot 36$ & $0 \cdot 37$ \\
\hline $\mathrm{Zn}(\mathrm{g} / \mathrm{d})$ & 10 & 8,14 & 13 & 10,17 & $0 \cdot 34$ & $0 \cdot 22$ & $0 \cdot 38$ \\
\hline $\operatorname{Mg}(g / d)$ & 215 & 177,279 & 310 & 244,375 & $0 \cdot 30$ & $0 \cdot 14$ & $0 \cdot 34$ \\
\hline $\mathrm{Cu}(\mathrm{mg} / \mathrm{d})$ & $1 \cdot 0$ & $1 \cdot 0,1 \cdot 3$ & $1 \cdot 6$ & $1 \cdot 1,2 \cdot 2$ & $0 \cdot 11$ & 0.05 & $0 \cdot 12$ \\
\hline Folic acid $(\mu \mathrm{g} / \mathrm{d})$ & 162 & 109,233 & 273 & 194,346 & $0 \cdot 21$ & 0.20 & 0.24 \\
\hline Vitamin A (IU) & 5910 & 2951,11245 & 11585 & 6756,15814 & 0.29 & 0.33 & $0 \cdot 32$ \\
\hline Vitamin A (RE) & 704 & 414,1514 & 1483 & 885,2275 & $0 \cdot 30$ & 0.33 & 0.34 \\
\hline Thiamin (mg/d) & $1 \cdot 3$ & $1 \cdot 0,1 \cdot 6$ & $1 \cdot 7$ & $1 \cdot 3,2 \cdot 6$ & $0 \cdot 28$ & -0.03 & $0 \cdot 31$ \\
\hline Riboflavin (mg/d) & $1 \cdot 7$ & $1 \cdot 0,2 \cdot 2$ & $2 \cdot 2$ & $1 \cdot 6,3 \cdot 1$ & 0.34 & 0.34 & $0 \cdot 38$ \\
\hline $\operatorname{Niacin}(\mathrm{mg} / \mathrm{d})$ & 17 & 17,22 & 21 & 16,29 & $0 \cdot 35$ & $0 \cdot 36$ & 0.41 \\
\hline Vitamin $C(\mathrm{mg} / \mathrm{d})$ & 65 & 23,135 & 182 & 92,318 & $0 \cdot 18$ & 0.29 & $0 \cdot 20$ \\
\hline Vitamin E (mg/d) & 4 & 3,7 & 7 & 6,10 & $0 \cdot 30$ & $0 \cdot 17$ & $0 \cdot 33$ \\
\hline Vitamin $B_{6}(\mathrm{mg} / \mathrm{d})$ & $1 \cdot 0$ & $0 \cdot 7,1 \cdot 3$ & $1 \cdot 2$ & $0 \cdot 8,1 \cdot 7$ & $0 \cdot 32$ & $0 \cdot 18$ & $0 \cdot 37$ \\
\hline Fibre $(g / d)$ & 22 & 15,27 & 32 & 23,38 & $0 \cdot 37$ & $0 \cdot 19$ & $0 \cdot 41$ \\
\hline SFA $(g / d)$ & 21 & 15,27 & 30 & 19,38 & $0 \cdot 31$ & 0.02 & $0 \cdot 34$ \\
\hline Cholesterol (g/d) & 238 & 173,310 & 265 & 164,348 & $0 \cdot 17$ & 0.07 & $0 \cdot 20$ \\
\hline Mean & - & & - & & $0 \cdot 27$ & $0 \cdot 16$ & $0 \cdot 32$ \\
\hline
\end{tabular}

$24 \mathrm{hR}, 24 \mathrm{~h}$ dietary recall, IQR, interquartile range, $\mathrm{RE}$, retinol equivalents. ${ }^{*}$ Nutrient intake values were natural log-transformed and energy-adjusted. tNutrient intake values were natural log-transformed only.

The joint classification between methods is presented in Table 3. Concerning estimated nutrient intakes for the first two trimesters of pregnancy, $32 \%$ of women were classified into the same quartile by both methods; approximately $72 \%$ were classified into the same or adjacent quartiles, while only $7 \cdot 8 \%$ of the women were classified into opposite quartiles. For estimated nutrient intakes for the gestational period as a whole, approximately $33 \%$ of pregnant women were classified into the same quartile, $71 \%$ into the same or adjacent quartiles and $7 \cdot 8 \%$ into opposite quartiles according to the two different methods of assessing food consumption. However, lower agreements into the lowest and highest quartiles were found for both periods of pregnancy.

\section{Discussion}

The FFQ used in the present study was the first developed and validated specifically for pregnant women in Brazil. 
Table 3 Joint classification into quartiles for nutrient intakes estimated by the two methods and quadratic kappa statistic in pregnant women - Ribeirão Preto, São Paulo, Brazil, 2009

\begin{tabular}{|c|c|c|c|c|c|c|c|c|c|c|c|c|}
\hline \multirow[b]{2}{*}{ Nutrient ${ }^{\star}$} & \multicolumn{2}{|c|}{$\begin{array}{l}\text { Same } \\
\text { quartile }\end{array}$} & \multicolumn{2}{|c|}{$\begin{array}{l}\text { Lowest } \\
\text { quartile }\end{array}$} & \multicolumn{2}{|c|}{$\begin{array}{l}\text { Highest } \\
\text { quartile }\end{array}$} & \multicolumn{2}{|c|}{$\begin{array}{c}\text { Same+adjacent } \\
\text { quartiles }\end{array}$} & \multicolumn{2}{|c|}{$\begin{array}{l}\text { Opposite } \\
\text { quartile }\end{array}$} & \multirow[b]{2}{*}{ Quadratic $\kappa$} & \multirow[b]{2}{*}{$P$ value } \\
\hline & $n$ & $\%$ & $n$ & $\%$ & $n$ & $\%$ & $n$ & $\%$ & $n$ & $\%$ & & \\
\hline & \multicolumn{12}{|c|}{ Estimated nutrient intakes and accuracy of FFQ for first two trimesters of pregnancy $(n 88)$} \\
\hline Energy $(\mathrm{kJ} / \mathrm{d}) \dagger$ & 31 & $35 \cdot 2$ & 9 & $10 \cdot 2$ & 5 & $5 \cdot 7$ & 71 & $80 \cdot 7$ & 6 & $7 \cdot 0$ & 0.31 & 0.03 \\
\hline Protein $(\mathrm{g} / \mathrm{d})$ & 22 & $25 \cdot 0$ & 9 & $10 \cdot 2$ & 6 & $7 \cdot 0$ & 59 & $67 \cdot 0$ & 3 & $3 \cdot 5$ & 0.23 & $<0.0001$ \\
\hline Lipids $(\mathrm{g} / \mathrm{d})$ & 31 & $35 \cdot 2$ & 10 & $11 \cdot 3$ & 6 & $7 \cdot 0$ & 58 & $66 \cdot 0$ & 9 & $10 \cdot 2$ & $0 \cdot 12$ & $<0.0001$ \\
\hline Carbohydrate $(\mathrm{g} / \mathrm{d})$ & 34 & $38 \cdot 6$ & 12 & $13 \cdot 6$ & 7 & $8 \cdot 0$ & 68 & $77 \cdot 2$ & 6 & $7 \cdot 0$ & 0.26 & $<0.0001$ \\
\hline $\mathrm{Ca}(\mathrm{g} / \mathrm{d})$ & 39 & $44 \cdot 3$ & 10 & $11 \cdot 3$ & 9 & $10 \cdot 2$ & 71 & $80 \cdot 7$ & 4 & $4 \cdot 5$ & 0.45 & $<0.0001$ \\
\hline $\mathrm{Fe}(\mathrm{mg} / \mathrm{d})$ & 31 & $35 \cdot 2$ & 11 & $12 \cdot 5$ & 4 & $4 \cdot 5$ & 63 & $71 \cdot 6$ & 8 & $9 \cdot 0$ & $0 \cdot 21$ & $<0.0001$ \\
\hline$K(g / d)$ & 24 & $27 \cdot 3$ & 12 & $13 \cdot 6$ & 9 & $10 \cdot 2$ & 62 & $70 \cdot 5$ & 6 & $7 \cdot 0$ & $0 \cdot 22$ & $0 \cdot 4$ \\
\hline Vitamin A (IU) & 27 & $30 \cdot 7$ & 10 & $11 \cdot 3$ & 7 & $8 \cdot 0$ & 62 & $70 \cdot 5$ & 11 & $12 \cdot 5$ & $0 \cdot 12$ & $<0.0001$ \\
\hline Vitamin A (RE) & 29 & $33 \cdot 0$ & 9 & 10.2 & 7 & $8 \cdot 0$ & 66 & $75 \cdot 0$ & 7 & $8 \cdot 0$ & $0 \cdot 27$ & $<0.0001$ \\
\hline Thiamin (mg/d) & 20 & $22 \cdot 7$ & 6 & $7 \cdot 0$ & 5 & $5 \cdot 7$ & 55 & $62 \cdot 5$ & 11 & $12 \cdot 5$ & 0.009 & $<0.0001$ \\
\hline Riboflavin (mg/d) & 30 & $34 \cdot 1$ & 11 & $12 \cdot 5$ & 8 & $9 \cdot 0$ & 68 & $77 \cdot 2$ & 6 & $7 \cdot 0$ & $0 \cdot 32$ & 0.6 \\
\hline $\operatorname{Niacin}(\mathrm{mg} / \mathrm{d})$ & 29 & $33 \cdot 0$ & 10 & $11 \cdot 3$ & 7 & $8 \cdot 0$ & 67 & $76 \cdot 1$ & 6 & $7 \cdot 0$ & 0.31 & $0 \cdot 11$ \\
\hline Vitamin $B_{6}(\mathrm{mg} / \mathrm{d})$ & 25 & $28 \cdot 5$ & 7 & $8 \cdot 0$ & 6 & $7 \cdot 0$ & 63 & $71 \cdot 6$ & 6 & $7 \cdot 0$ & 0.23 & 0.03 \\
\hline Vitamin C (mg/d) & 29 & $33 \cdot 0$ & 11 & $12 \cdot 5$ & 10 & $11 \cdot 3$ & 64 & $72 \cdot 7$ & 3 & $3 \cdot 5$ & 0.33 & $<0.0001$ \\
\hline Dietary fibre (g/d) & 37 & $42 \cdot 0$ & 8 & $9 \cdot 0$ & 7 & $8 \cdot 0$ & 71 & $80 \cdot 7$ & 2 & $2 \cdot 3$ & 0.49 & 0.02 \\
\hline Folic acid $(\mu \mathrm{g} / \mathrm{d})$ & 27 & $30 \cdot 7$ & 11 & $12 \cdot 5$ & 9 & $10 \cdot 2$ & 68 & $77 \cdot 2$ & 5 & $5 \cdot 7$ & 0.33 & 0.07 \\
\hline Vitamin E (mg/d) & 35 & $40 \cdot 0$ & 8 & $9 \cdot 0$ & 7 & $8 \cdot 0$ & 71 & $80 \cdot 7$ & 7 & $8 \cdot 0$ & 0.32 & $<0.0001$ \\
\hline $\mathrm{Zn}(\mathrm{g} / \mathrm{d})$ & 31 & $35 \cdot 2$ & 7 & $8 \cdot 0$ & 10 & $11 \cdot 3$ & 65 & $73 \cdot 8$ & 4 & $4 \cdot 5$ & 0.33 & 0.97 \\
\hline $\mathrm{Mg}(\mathrm{mg} / \mathrm{d})$ & 26 & $29 \cdot 5$ & 12 & $13 \cdot 6$ & 7 & $8 \cdot 0$ & 61 & $69 \cdot 3$ & 5 & $5 \cdot 7$ & 0.23 & $0 \cdot 15$ \\
\hline $\mathrm{Cu}(\mathrm{mg} / \mathrm{d})$ & 25 & $28 \cdot 5$ & 9 & $10 \cdot 2$ & 9 & $10 \cdot 2$ & 66 & $75 \cdot 0$ & 9 & $10 \cdot 2$ & $0 \cdot 21$ & $<0.0001$ \\
\hline SFA (g/d) & 25 & $28 \cdot 5$ & 8 & $9 \cdot 0$ & 6 & $7 \cdot 0$ & 55 & $62 \cdot 5$ & 11 & $12 \cdot 5$ & 0.08 & 0.05 \\
\hline Cholesterol (g/d) & 27 & $30 \cdot 7$ & 6 & $8 \cdot 3$ & 7 & $8 \cdot 0$ & 64 & $72 \cdot 7$ & 7 & $8 \cdot 0$ & 0.23 & 0.78 \\
\hline \multirow[t]{2}{*}{ Mean } & 28 & $31 \cdot 7$ & 9 & $10 \cdot 6$ & 7 & $8 \cdot 2$ & 63 & $72 \cdot 0$ & 6 & $7 \cdot 8$ & $0 \cdot 24$ & - \\
\hline & \multicolumn{12}{|c|}{ Estimated nutrient intake and accuracy of FFQ for whole gestational period ( $n$ 73) } \\
\hline Energy $(\mathrm{kJ} / \mathrm{d}) \dagger$ & 28 & $38 \cdot 8$ & 10 & $13 \cdot 7$ & 9 & $12 \cdot 3$ & 63 & $87 \cdot 5$ & 1 & $1 \cdot 4$ & 0.30 & 0.4800 \\
\hline Protein $(\mathrm{g} / \mathrm{d})$ & 24 & $33 \cdot 3$ & 5 & $7 \cdot 0$ & 8 & $11 \cdot 1$ & 52 & $72 \cdot 2$ & 4 & $5 \cdot 5$ & 0.28 & $0 \cdot 13$ \\
\hline Lipids (g/d) & 20 & $27 \cdot 7$ & 7 & $9 \cdot 7$ & 4 & $5 \cdot 5$ & 51 & $70 \cdot 8$ & 5 & $7 \cdot 0$ & $0 \cdot 21$ & 0.45 \\
\hline Carbohydrate $(\mathrm{g} / \mathrm{d})$ & 23 & $32 \cdot 0$ & 6 & $8 \cdot 3$ & 8 & $11 \cdot 1$ & 51 & $70 \cdot 8$ & 4 & $5 \cdot 5$ & 0.25 & 0.63 \\
\hline $\mathrm{Ca}(\mathrm{g} / \mathrm{d})$ & 22 & $30 \cdot 5$ & 6 & $8 \cdot 3$ & 8 & $11 \cdot 1$ & 55 & $76 \cdot 4$ & 5 & $7 \cdot 0$ & $0 \cdot 30$ & 0.4454 \\
\hline $\mathrm{Fe}(\mathrm{mg} / \mathrm{d})$ & 20 & $27 \cdot 7$ & 7 & $9 \cdot 7$ & 3 & $4 \cdot 1$ & 46 & $63 \cdot 8$ & 8 & $11 \cdot 1$ & 0.05 & $<0.0001$ \\
\hline$K(g / d)$ & 30 & $41 \cdot 6$ & 10 & $13 \cdot 7$ & 8 & $11 \cdot 1$ & 61 & $84 \cdot 7$ & 7 & $9 \cdot 7$ & 0.50 & 0.2668 \\
\hline Vitamin A (IU) & 28 & $38 \cdot 8$ & 8 & $11 \cdot 1$ & 9 & $12 \cdot 3$ & 52 & $72 \cdot 2$ & 4 & $5 \cdot 5$ & $0 \cdot 31$ & 0.4259 \\
\hline Vitamin A (RE) & 26 & $36 \cdot 1$ & 7 & $9 \cdot 7$ & 6 & $8 \cdot 3$ & 52 & $72 \cdot 2$ & 8 & $11 \cdot 1$ & $0 \cdot 18$ & 0.0034 \\
\hline Thiamin (mg/d) & 17 & $23 \cdot 6$ & 5 & $7 \cdot 0$ & 1 & $1 \cdot 4$ & 44 & $61 \cdot 1$ & 11 & $15 \cdot 2$ & -0.07 & 0.2779 \\
\hline Riboflavin (mg/d) & 37 & $51 \cdot 4$ & 9 & $12 \cdot 3$ & 11 & $15 \cdot 2$ & 56 & $77 \cdot 7$ & 3 & $4 \cdot 1$ & 0.45 & 0.0087 \\
\hline $\operatorname{Niacin}(\mathrm{mg} / \mathrm{d})$ & 23 & $32 \cdot 0$ & 9 & $12 \cdot 3$ & 6 & $8 \cdot 3$ & 55 & $76 \cdot 4$ & 2 & $2 \cdot 7$ & 0.38 & $<0.0001$ \\
\hline Vitamin $B_{6}(\mathrm{mg} / \mathrm{d})$ & 23 & $32 \cdot 0$ & 7 & $9 \cdot 7$ & 8 & $11 \cdot 1$ & 46 & $63 \cdot 8$ & 5 & $7 \cdot 0$ & $0 \cdot 15$ & $<0.0001$ \\
\hline Vitamin $C(\mathrm{mg} / \mathrm{d})$ & 22 & $30 \cdot 5$ & 5 & $7 \cdot 0$ & 7 & $9 \cdot 7$ & 51 & $70 \cdot 8$ & 5 & $7 \cdot 0$ & 0.23 & 0.0489 \\
\hline Dietary fibre $(\mathrm{g} / \mathrm{d})$ & 27 & $37 \cdot 5$ & 7 & $9 \cdot 7$ & 7 & $9 \cdot 7$ & 52 & $72 \cdot 2$ & 7 & $9 \cdot 7$ & 0.22 & 0.0994 \\
\hline Folic acid $(\mu \mathrm{g} / \mathrm{d})$ & 22 & $30 \cdot 5$ & 8 & $11 \cdot 1$ & 5 & $7 \cdot 0$ & 51 & $70 \cdot 8$ & 4 & $5 \cdot 5$ & 0.34 & 0.2896 \\
\hline Vitamin E (mg/d) & 22 & $30 \cdot 5$ & 7 & $9 \cdot 7$ & 4 & $5 \cdot 5$ & 53 & $73 \cdot 6$ & 7 & $9 \cdot 7$ & $0 \cdot 21$ & 0.0833 \\
\hline $\operatorname{Zn}(g / d)$ & 21 & $29 \cdot 1$ & 5 & $7 \cdot 0$ & 7 & $9 \cdot 7$ & 51 & $70 \cdot 8$ & 6 & $8 \cdot 3$ & $0 \cdot 20$ & 0.6448 \\
\hline $\mathrm{Mg}(\mathrm{mg} / \mathrm{d})$ & 22 & $30 \cdot 5$ & 8 & $11 \cdot 1$ & 6 & $8 \cdot 3$ & 57 & $79 \cdot 0$ & 5 & $7 \cdot 0$ & 0.33 & 0.5739 \\
\hline $\mathrm{Cu}(\mathrm{mg} / \mathrm{d})$ & 19 & $26 \cdot 4$ & 7 & $9 \cdot 7$ & 4 & $5 \cdot 5$ & 45 & $62 \cdot 5$ & 4 & $5 \cdot 5$ & $0 \cdot 14$ & 0.0954 \\
\hline $\operatorname{SFA}(g / d)$ & 24 & $33 \cdot 3$ & 8 & $11 \cdot 1$ & 7 & $9 \cdot 7$ & 50 & $69 \cdot 4$ & 4 & $5 \cdot 5$ & 0.25 & $0 \cdot 8446$ \\
\hline Cholesterol (g/d) & 23 & $32 \cdot 0$ & 7 & $9 \cdot 7$ & 6 & $8 \cdot 3$ & 51 & $70 \cdot 8$ & 8 & $11 \cdot 1$ & $0 \cdot 15$ & $0 \cdot 2486$ \\
\hline Mean & 23 & $32 \cdot 6$ & 7 & $10 \cdot 0$ & 6 & $8 \cdot 2$ & 51 & $71 \cdot 0$ & 5 & $7 \cdot 8$ & 0.23 & - \\
\hline
\end{tabular}

$\mathrm{RE}$, retinol equivalents.

In addition, few validation studies of FFQ for pregnant women have assessed the accuracy of the method for estimating nutrient intakes during different periods of pregnancy. The FFQ showed acceptable accuracy $(r>0 \cdot 35)$, based on energy-adjusted and deattenuated Pearson's correlation coefficient, for estimating $\mathrm{Ca}, \mathrm{K}, \mathrm{Zn}, \mathrm{Mg}$, Fe, fibre, SFA, vitamin A, thiamin, riboflavin, niacin, vitamin $\mathrm{C}$ and folic acid for the first two trimesters of pregnancy. Considering the gestational period as a whole, acceptable correlation was found for energy, carbohydrate, protein, lipids, $\mathrm{Fe}, \mathrm{K}, \mathrm{Zn}$, fibre, vitamin $\mathrm{B}_{6}$, riboflavin and niacin. A high proportion of study participants $(\geq 70 \%)$ were categorized into the same or adjacent quartiles for estimated energy, carbohydrate, Ca, K, fibre, Zn, cholesterol, vitamin A, riboflavin, niacin, vitamin $\mathrm{C}$, vitamin $\mathrm{E}$ and folic acid in both gestational periods.

In a study involving Finnish pregnant women, Erkkola et $a l^{(15)}$ found estimated nutrient intakes measured by an FFQ to be $30-40 \%$ higher than mean values calculated using food records. According to Robinson et al. ${ }^{(11)}$, 
higher consumption reported by FFQ may result from the bigger portions used in the FFQ or be due to overreporting of intake frequency. To develop the FFQ used in the present study, nutrient intake was adjusted by energy and within-person variation ${ }^{(17)}$ in order to reduce the risk of the instrument overestimating intake. Although the food list of the FFQ assessed is very similar to the lists used in other FFQ devised for use in Brazil ${ }^{(29,30)}$, portion sizes were consistently larger.

The values of Pearson's correlation coefficient in the present study were, on average, lower for macronutrients, although satisfactory correlation was confirmed for the majority of the nutrients of interest in this investigation. Other studies in pregnant women employing Pearson's correlation coefficient for FFQ validation have also found lower-than-expected correlation coefficients. For instance, in a study of eighty US pregnant women using three replicates of $24 \mathrm{hR}$, Forsythe and Gage ${ }^{(10)}$ found Pearson's correlation coefficients ranging from 0.42 for vitamin $B_{12}$ to 0.46 for Fe. In a study conducted among pregnant women in Brazil, Giacomello et al. ${ }^{(14)}$ identified Pearson's correlation coefficients of between $0 \cdot 01$ for saturated fats and 0.47 for $\mathrm{Ca}$, taking two $48 \mathrm{hR}$ as the reference method. The low correlation coefficients seen in validation studies of FFQ for pregnant women can be partially explained by the high within-person variability in estimated energy and nutrient intakes during this phase of life, especially when few replications of $24 \mathrm{hR}$ or records are taken as the reference method $^{(12)}$.

After adjustments for energy, higher correlations between the dietary methods were found for protein, lipids, K, Zn, Mg and fibre estimates for the first 24 weeks of pregnancy. Considering the gestational period as a whole, higher correlations were verified for $\mathrm{Ca}, \mathrm{K}$, vitamin $\mathrm{A}$, niacin and vitamin $\mathrm{C}$. The improvement in correlations after energy adjustment might be related to the correction of correlated errors on nutrient and energy estimates ${ }^{(26,27)}$.

International validation studies of questionnaires developed and validated for use in pregnant women suggest this method is a valuable tool for assessing the dietary intake of pregnant women ${ }^{(12,15,16)}$, evidenced by acceptable correlation coefficient values ${ }^{(8)}$. Current recommendations advise against the use of Pearson's correlation coefficient alone for assessing agreement between two methods, instead suggesting its use in association with other analysis techniques, such as categorization into intake levels, weighted $\kappa$ statistics and the Bland-Altman method $^{(6)}$. However, Bland-Altman analysis requires a minimum sample size of 100 individuals.

In the present study, the percentage of pregnant women classified into the same consumption quartile (32-33\%) by both the FFQ and $24 \mathrm{hR}$, and into opposite $(7 \cdot 8 \%)$ quartiles, was similar to that reported by other validation studies of FFQ for Portuguese pregnant women ${ }^{(31)}$. The agreement between the methods observed in the present study was also similar to that seen in a previous study conducted in Brazil assessing the accuracy of an FFQ developed for adults in estimating nutrient intakes among pregnant women, in which $30 \%$ of pregnant women were classified into the same quartile of estimated nutrients and $8 \%$ into opposite quartiles. The proportion of agreement in the same or adjacent quartiles observed in the present study $(\sim 72 \%)$ was similar to that reported in an FFQ validation study performed among pregnant Finnish women ${ }^{(15)}$ in which $69 \%$ of the women were classified into the same or adjacent quintiles and 5\% into opposite quintiles.

The quadratic weighted $\kappa$ value found in the present study was low, in line with findings of a previous FFQ validation study conducted in Brazil reporting $\kappa$ values ranging from 0.39 for vitamin $\mathrm{C}$ to 0.06 for $\mathrm{Cu}^{(14)}$.

According to Beaton ${ }^{(32)}$, the fact that the FFQ under- or overestimates nutrients is not an issue in epidemiological studies aimed at identifying diet-disease associations provided that the classification of individuals by intake levels is acceptable. Given this premise, if the aim of the FFQ is to categorize individuals into quartiles of intake then the FFQ used in the present study can be deemed effective since, for the majority of nutrients, $70 \%$ or more of the pregnant women were categorized into the same or adjacent quartiles. Therefore, these results show that the FFQ can be employed for this purpose. However, low proportions of women were correctly classified into the lowest and highest quartiles.

Despite the limitation of using a fixed list of foods as well as standardized food portions, factors which could compromise accuracy of nutrient estimates when employing the FFQ, the high number of $24 \mathrm{hR}$ or food records replicates needed to achieve an accurate estimate of nutrients during pregnancy can render the assessment of dietary intake impractical in this population group in epidemiological studies. Based on the data of the present study, considering within-person variance data and accuracy values of approximately 20\%, four $24 \mathrm{hR}$ would be needed to accurately estimate energy intake, three for carbohydrates, eighteen for lipids, seven for protein, twenty-four for $\mathrm{Ca}$ and nine for fibre (data not shown).

The main limitation of the current study was the use of only a small number of $24 \mathrm{hR}$ as the 'gold standard' for estimating nutrients of the usual dietary intake of pregnant women. The FFQ and $24 \mathrm{hR}$ have similar magnitudes of error for measures such as memory bias, perhaps constituting another limitation of the study. However, given the low literacy and motivation of participants in the present study, multiple $24 \mathrm{hR}$ was considered the most appropriate reference method. Moreover, a minimum sample size of 100 individuals is recommended for analyses using the Bland-Altman method. The initial sample of the present study numbered 103 pregnant women. However, after losses to follow-up, only eighty-eight pregnant women continued to the second assessment of 
the study whereas seventy-two remained for the third assessment, restraining the use of Bland-Altman analysis in the present study ${ }^{(33)}$.

The sample of the present study was pregnant women attended by the national health-care system in Ribeirão Preto, São Paulo State. According to the National Household Survey, $74 \%$ of Brazilians are users of this health service in the country, and share similar characteristics on schooling level and economic classes ${ }^{(34)}$. The applicability of the tested FFQ in studies conducted among pregnant women in Brazil will be dependent on nutrients of interest to the investigation and on the similarity of food habits, which might diverge according to the region of the country. Therefore, calibration studies are recommended prior to utilization of the FFQ in different populations.

\section{Conclusions}

The FFQ developed was assessed and validated for pregnant women. The results showed acceptable correlation of the instrument for estimating intakes of $\mathrm{Ca}, \mathrm{K}$, $\mathrm{Zn}, \mathrm{Mg}$, fibre, vitamin $\mathrm{C}$, niacin and folic acid for the first two trimesters of pregnancy, and for energy, lipids, protein, carbohydrate, $\mathrm{Fe}, \mathrm{K}, \mathrm{Zn}$, fibre, vitamin $\mathrm{B}_{6}$, riboflavin and niacin taking the gestational period as a whole. Furthermore, the FFQ proved a satisfactory method of categorizing estimated nutrients of interest in the diet of the pregnant women assessed.

\section{Acknowledgements}

Source of funding: The project received financial support from the research funding body Coordenação de Aperfeiçoamento de Pessoal de Nível Superior (CAPES) and from the Fundação de Apoio ao Ensino, Pesquisa e Assistência do Hospital das Clínicas da Faculdade de Medicina de Ribeirão Preto da Universidade de São Paulo (FAEPA). Conflicts of interest: The authors declare that they have no conflicts of interest. Authors' contribution: D.S.S. is coordinator of the study. All authors have carried out the study and interpreted the results. P.B. wrote the first draft of the manuscript. All authors read and approved the final manuscript.

\section{References}

1. Meltzer HM, Brantsaeter AL, Nilsen RM et al. (2011) Effect of dietary factors in pregnancy on risk of pregnancy complications: results from the Norwegian Mother and Child Cohort Study. Am J Clin Nutr 96, 6 Suppl., 1970S-1974S.

2. Oken E, Ning YI, Sheril LL et al. (2007) Diet during pregnancy and risk of preeclampsia or gestational hypertension. Ann Epidemiol 17, 663-668.

3. Lim C, Yii MF \& Cheng NCL (2009) The role of micronutrients in pregnancy. Aust Fam Physician 38, 980-984.
4. Yajnik CS \& Deshmukh US (2008) Maternal nutrition, intrauterine programming and consequential risks in the offspring. Rev Endocr Metab Disord 9, 203-211.

5. Fall C (2009) Maternal nutrition: effects on health in the next generation. Indian J Med Res 130, 593-599.

6. Cade J, Thompson R, Burley V et al. (2002) Development, validation and utilization of food-frequency questionnaires a review. Public Health Nutr 5, 567-587.

7. Chen L, Hu FB, Yeung E et al. (2009) Prospective study of pre-gravid sugar-sweetened beverage consumption and the risk of gestational diabetes mellitus. Diabetes Care $\mathbf{3 2}$, 2236-2241.

8. Brown JE, Buzzard IM, Jacobs DR Jr et al. (1996) A food frequency questionnaire can detect pregnancy-related changes in diet. J Am Diet Assoc 96, 262-266.

9. Fawzi WW, Rifas-Shiman SL, Rich-Edwards JW et al. (2004) Calibration of a semi-quantitative food frequency questionnaire in early pregnancy. Ann Epidemiol 14, 754-762.

10. Forsythe HE \& Gage B (1994) Use of a multicultural foodfrequency questionnaire with pregnant and lactating women. Am J Clin Nutr 59, 1 Suppl., S203-S206.

11. Robinson S, Godfrey K, Osmond C et al. (1996) Evaluation of a food frequency questionnaire used to assess nutrient intakes in pregnant women. Eur J Clin Nutr 50, 302-308.

12. Baer HJ, Blum RE, Rockett RHH et al. (2005) Use of a food frequency questionnaire in American Indian and Caucasian pregnant women: a validation study. BMC Public Health 5, 135 .

13. Wei EK, Gardner J, Field AE et al. (1999) Validity of a food frequency questionnaire in assessing nutrient intakes of low-income pregnant women. Matern Child Health J 3, 241-246.

14. Giacomello A, Schimdt MI, Nunes MAA et al. (2008) Validação relativa de questionário de freqüência alimentar em gestantes usuárias de serviços do Sistema Único de Saúde em dois municípios no Rio Grande do Sul, Brasil. Rev Bras Matern Infant 8, 445-454.

15. Erkkola M, Karppinen M, Javanainen J et al. (2001) Validity and reproducibility of a food frequency questionnaire for pregnant Finnish women. Am J Epidemiol 154, 466-476.

16. Mouratidou T, Ford F \& Frazer RB (2006) Validation of a food-frequency questionnaire for use in pregnancy. Public Health Nutr 9, 515-522.

17. Oliveira T, Marquitti FD, Carvalhaes MABL et al. (2010) Desenvolvimento de um Questionário Quantitativo de Freqüência Alimentar (QQFA) para gestantes usuárias de unidades básicas de saúde de Ribeirão Preto, São Paulo, Brasil. Cad Saude Publica 26, 2296-2306.

18. Instituto Brasileiro de Geografia e Estatística (2011) Censo Demográfico 2010. Rio de Janeiro: IBGE; available at http://www.ibge.gov.br/home/estatistica/populacao/censo 2010/sinopse.pdf

19. Institute of Medicine (2009) Weight Gain During Pregnancy: Reexamining the Guidelines. Washington, DC: National Academy Press.

20. Associação Brasileira de Empresa de Pesquisas (2008) Dados com base no levantamento socioeconômico 2006 e 2007. http://www.abep.org (accessed January 2008).

21. Johnson RK, Soultanakis RP \& Matthews DE (1998) Literacy and body fatness are associated with underreporting of energy intake in US low income women using the multiplepass 24 hours recall: a doubly labeled water study. J Am Diet Assoc 98, 1136-1140.

22. Pinheiro ABV, Lacerda EMA, Benzecry EH et al. (2005) Tabela para avaliação de consumo alimentar em medidas caseiras, 5th ed. São Paulo: Editora Atheneu.

23. Fisberg RF \& Slater B (2002) Manual de receitas e medidas caseiras para cálculo de inquéritos alimentares: manual elaborado para auxiliar o processamento de inquéritos alimentares. São Paulo: Signus. 
24. Núcleo de Estudos e Pesquisa em Alimentação (2006) Tabela Brasileira de composição de alimentos - TACO, Versão 2, 2nd ed. Campinas: Universidade Estadual de Campinas.

25. US Department of Agriculture (2001) Nutrient Database for Standard Reference. Beltsville, MD: Agricultural Research Service, USDA; available at http://www.usda.gov

26. Willett WC \& Stampfer MJ (1996) Total energy intake: implications for epidemiologic analyses. Am J Epidemiol 124, 17-27.

27. Willett WC (1998) Nutritional Epidemiology, 2nd ed. New York: Oxford University Press.

28. Brenner H \& Kliebsch U (1996) Dependence of weighted kappa coefficients on the number of categories. Epidemiology 7, 199-202.

29. Cardoso MA \& Stocco PR (2000) Desenvolvimento de um questionário quantitativo de freqüência alimentar em imigrantes japoneses e seus descendentes residentes em São Paulo, Brasil. Cad Saude Publica 16, 107-114.
30. Sichieri R \& Everhart JE (1998) Validity of a Brazilian food frequency questionnaire against dietary recalls and estimated energy intake. Nutr Res 18, 1649-1659.

31. Pinto E, Severo M, Correia S et al. (2010) Validity and reproducibility of a semi quantitative food frequency questionnaire for use among Portuguese pregnant women. Matern Child Nutr 6, 105-119.

32. Beaton GH (1991) Interpretation of results from diet history studies. In The Diet History Method, pp. 15-38 [L Kohlmeier, editor]. London: Smith-Gordon.

33. Bland JM \& Altman DG (1995) Comparing methods of measurement: why plotting difference against standard method is misleading. Lancet 345, 1085-1087.

34. Instituto Brasileiro de Geografia e Estatística (2009) Pesquisa nacional por amostragem de domicilios: 2008. Rio de Janeiro: IBGE; http://www.ibge.gov.br/home/ presidencia/noticias/noticia_visualiza.php?id_noticia $=1455 \&$ id_pagina 